\title{
The surgical anatomy of the lateral femoral cutaneous nerve in the inguinal region: a meta-analysis
}

\author{
K. A. Tomaszewski ${ }^{1,2} \cdot$ P. Popieluszko ${ }^{1,2} \cdot$ B. M. Henry ${ }^{1,2} \cdot$ J. Roy ${ }^{1,2}$. \\ B. Sanna ${ }^{3}$ M. R. Kijek ${ }^{2}$ J. A. Walocha,
}

Received: 26 January 2016/ Accepted: 13 April 2016/Published online: 26 April 2016

(c) The Author(s) 2016. This article is published with open access at Springerlink.com

\begin{abstract}
Purpose Several variations in the anatomy and injury of the lateral femoral cutaneous nerve (LFCN) have been studied since 1885 . The aim of our study was to analyze the available data on the LFCN and find a true prevalence to help in the planning and execution of surgical procedures in the area of the pelvis, namely inguinal hernia repair.

Methods A search of the major medical databases was performed for LFCN anatomy. The anatomical data were collected and analyzed.

Results Twenty-four studies $(n=1,720)$ were included. The most common pattern of the LFCN exiting the pelvis was medial to the Sartorius as a single branch. When it exited in this pattern, it did so on average $1.90 \mathrm{~cm}$ medial to the anterior superior iliac spine (ASIS).

Conclusions The LFCN and its variations are important to consider especially during inguinal hernia repair, abdominoplasty, and iliac bone grafting. We suggest maintaining a distance of $3 \mathrm{~cm}$ or more from the ASIS when operating to prevent injury to the LFCN.
\end{abstract}

K.A. Tomaszewski and P. Popieluszko contributed equally to this study.

Electronic supplementary material The online version of this article (doi:10.1007/s10029-016-1493-7) contains supplementary material, which is available to authorized users.

K. A. Tomaszewski

krtomaszewski@gmail.com

1 International Evidence-Based Anatomy Working Group, Krakow, Poland

2 Department of Anatomy, Jagiellonian University Medical College, 12 Kopernika St, 31-034 Krakow, Poland

3 Faculty of Medicine and Surgery, University of Cagliari, Sardinia, Italy
Keywords Lateral femoral cutaneous nerve · Variations . Anatomy $\cdot$ Meta-analysis

\section{Introduction}

The lateral femoral cutaneous nerve (LFCN) of the thigh is normally a derivative of the posterior divisions of the L2 and L3 spinal nerves that travels through the pelvis heading towards the anterior superior iliac spine (ASIS). It then usually exits the lesser pelvis under the inguinal ligament (IL), anterior to the ASIS, bifurcates into an anterior and posterior division along the length of the thigh, and provides sensory innervation to the skin of the anterolateral and lateral aspects of the thigh [1].

The most common pathology associated with the LFCN is meralgia paresthetica, a condition entailing pain, a lack of sensation, or dysesthesia of the skin supplied by the LFCN [2]. Meralgia paresthetica may have numerous etiologies including pelvic inflammatory disease, pregnancy, various toxicities, tight clothing, and importantly, iatrogenic injuries from surgical procedures [3]. Detailed knowledge of variations in the pelvic exits and branching patterns of LFCN is crucial in diagnosing meralgia paresthetica, as well as avoiding injuries during surgical procedures, especially inguinal hernia repair. The incidence of nerve injury in laparoscopic inguinal hernia repair is about $2 \%$ [4]. Although relatively a small percentage, current studies estimate that close to 20 million hernia repairs are performed annually worldwide [5].

Variations in the LFCN's anatomy are common, with seven different points of exit from the pelvis having been observed [6]. Four of the variations can be classified into four zones relative to the ASIS, through which the LFCN may pass. These include medial to the ASIS and under the IL, medial to the ASIS and over the IL, directly over the 
ASIS, or lateral to the ASIS. Three more variations were found where the nerve passed through another tissue: the ASIS itself, the sartorius muscle, or the IL. Even when the nerve followed the anatomically normal exit pattern of medial to the ASIS and under the IL, the distance from the ASIS can vary, creating a zone where the LFCN can be encountered. Furthermore, five branching patterns were observed. These included the normal single nerve that would eventually bifurcate in the area of the thigh, bifurcation within the pelvis, bifurcation near the nerve's exit from the pelvis, trifurcation, and quadrification [3].

Though many original studies have been performed on the LFCN, to our knowledge there has yet to be a comprehensive meta-analysis of all the data reported from anatomical studies around the world. With a lack of such a study and the possible clinical implications of the anatomy of the LFCN in surgery in the area of the hip or pelvis, the aim of our study was to perform a comprehensive metaanalysis on the prevalence of the reported variations in the LFCN to provide evidence-based foundation of anatomical knowledge for surgeons preparing for interventions in the area of the pelvis.

\section{Materials and methods}

\section{Search strategy}

The major electronic databases (Pubmed, EMBASE, Scopus, ScienceDirect, Web of Science, SciELO, BIOSIS, and CNKI) were extensively searched through November 2015 in order to identify all potentially eligible articles for inclusion into the meta-analysis. The search strategy used for Pubmed is summarized in Table 1. No language or date restrictions were set. Furthermore, the references of included studies were searched to identify any other potentially eligible articles. Preferred Reporting Items for Systematic Reviews and Meta-analyses (PRISMA) guidelines were strictly followed by the authors (Supplement 1) [7].

\section{Eligibility assessment}

Two authors (P.P. and B.M.H.) independently assessed all articles potentially eligible for inclusion into the meta- analysis. All studies which reported relevant, extractable anatomical data on the LFCN were included. The exclusion criteria were (1) case reports and case series, letters to the editor, review articles and conference abstracts, (2) studies which reported incomplete or nonextractable data, (3) studies on patients with LFCN pathologies, such as entrapment of the LFCN, which may potentially be associated with variable anatomy of the nerve, and (4) animal studies. All studies which were written in languages not spoken fluently by any of the authors were translated by medical professionals, fluent in the original language of the publication and English. In the case of any inconsistencies during the study selection process, a consensus between all the reviewers was reached, after consulting with the authors of the original study if possible.

\section{Data extraction}

Three reviewers (P.P., B.M.H. and B.S.) independently extracted data from the studies included in the meta-analysis. Data on the study type, sample size, geographical location, prevalence of the various exits of the LFCN from the pelvis, prevalence of the branching patterns of the LFCN in the pelvis and in the region of the IL, and the mean distance of the LFCN from its point of exit to the ASIS were extracted. Authors of all articles containing discrepancies in the data were contacted by email for additional information when possible.

\section{Statistical analysis}

Statistical analysis was performed by P.P. and J.R. using MetaXL version 2.0 by EpiGear International Pty Ltd (Wilston, QLD, Australia) to calculate multi-categorical pooled prevalence estimates for the various courses and branching patterns of the LCFA [8]. The morphometric data was pooled into an analysis using Comprehensive Meta-Analysis version 3.0 by Biostat (Englewood, NJ, USA). All analyses were performed by using a random effects model.

Heterogeneity among the studies included in the metaanalysis was measured using the chi square test and Higgins $I^{2}$ statistic. A Cochran's $Q P$ value of $<0.10$ for the chi

Table 1 Search strategy for Pubmed

1 ((( “lateral femoral cutaneous nerve"[Title/Abstract]) OR "lateral cutaneous femoral nerve"[Title/Abstract]) OR "lateral cutaneous nerve of the thigh" [Title/Abstract]) OR "lateral cutaneous nerve of thigh" [Title/Abstract]) OR "nervus cutaneus femoris posterior" [Title/Abstract]

2 (()((()(((“anatomy”[Title/Abstract]) OR “anatomical”[Title/Abstract]) OR “variation”[Title/Abstract]) OR “variations”[Title/Abstract]) OR “distribution”[Title/Abstract]) OR “course"[Title/Abstract]) OR “division"[Title/Abstract]) OR "variant”[Title/Abstract]) OR

"variants" [Title/Abstract]) OR “morphology”[Title/Abstract]) OR “morphological”[Title/Abstract]) OR "variability”[Title/Abstract]

31 AND 2 
square test was considered to be an indicator of significant heterogeneity between studies [9]. For Higgins $I^{2}$, values of 0-40\% were considered as "might not be important"; 30-60\% "might indicate moderate heterogeneity"; 50-90\% "may indicate substantial heterogeneity"; and 75-100 \% "may represent considerable heterogeneity" [9].

Subgroup analysis by type of study, geographical distribution, side (left vs. right) and/or a sensitivity analysis inclusive of studies with a number of lower limbs $\geq 100$, was conducted to probe potential sources of heterogeneity. Confidence intervals were used to determine statistically significant differences between two or more groups. In the case of overlapping confidence intervals, the differences were not considered statistically significant [8].

\section{Results}

\section{Study identification}

The study identification process is summarized in Fig. 1. All major electronic databases were extensively searched to identify 196 potentially eligible articles. A further four articles were added by searching the references of all articles included in the meta-analysis. Fifty-three articles were assessed using full text for potential eligibility, of which 28 were deemed ineligible and excluded for being case studies, reviews and lacking relevant, extractable data. Twenty-four articles were finally included into the metaanalysis.

\section{Characteristics of included studies}

The characteristics of included studies are presented in Table 2. A total of 25 studies ( $n=1,720$ lower limbs) were included into the meta-analysis [1, 3, 6, 10-32]. The dates of the included studies ranged from 1997 to 2015 and demonstrated a wide geographical distribution. Most studies were performed on cadavers, except the studies by Bodner et al. [10] and Zhu et al. [11] who used ultrasound and Watson et al. [12] who used magnetic resonance imaging. The study by Damarey et al. [13] included both cadaveric and imaging (ultrasound) modalities, and were thus for the purposes of this statistical analysis, consider as separate, independent samples.

\section{Exits of the lateral femoral cutaneous nerve from the pelvis}

A total of 18 studies ( $n=1,473$ lower limbs) reported data on where the LFCN exits in the pelvis. From our review, we identified seven types of exits from the pelvis: medial to

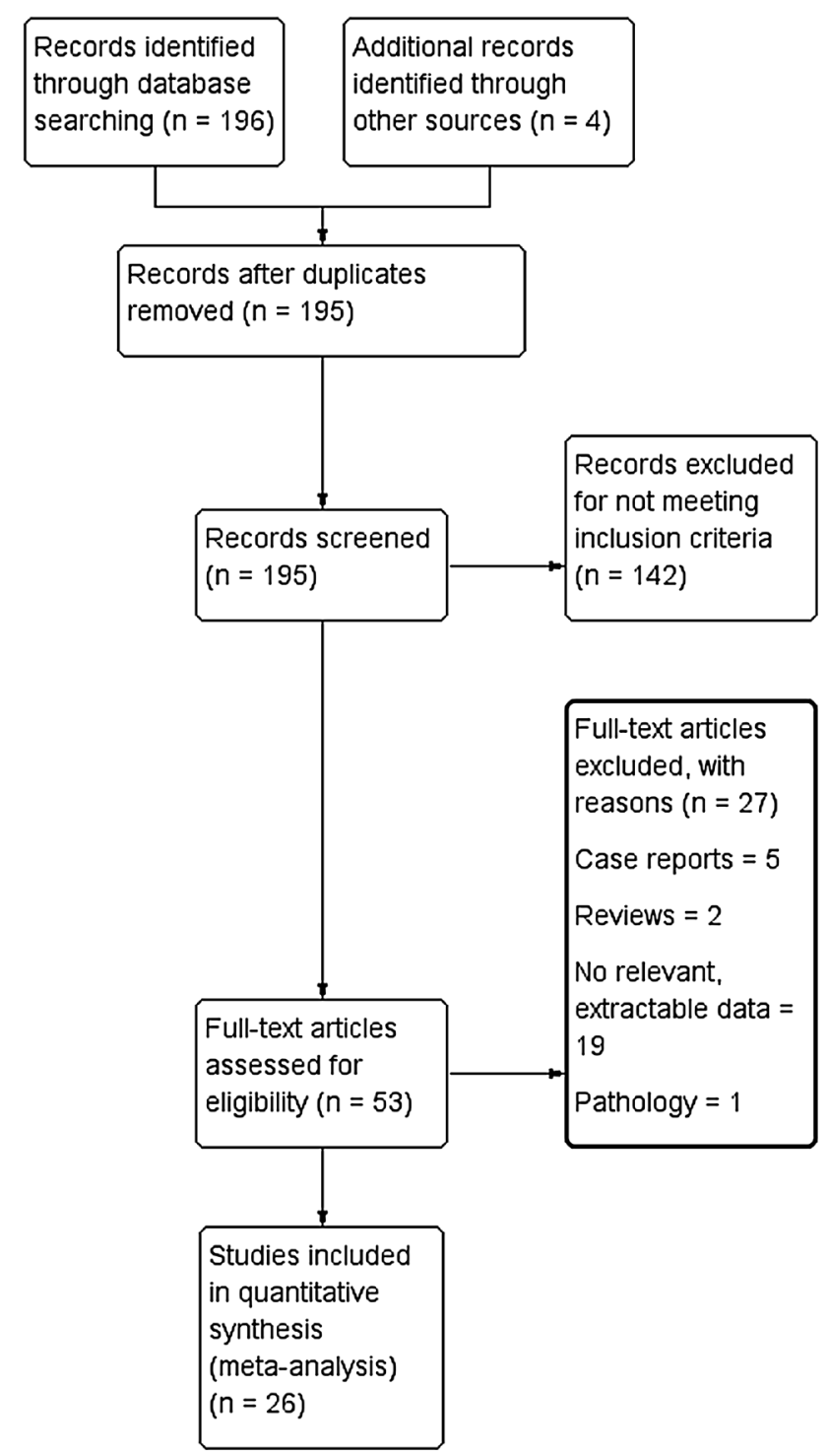

Fig. 1 PRISMA flowchart of studies included in the meta-analysis

the ASIS, through the IL, over the IL, directly over the ASIS, lateral to the ASIS, through the ASIS, and through the sartorius muscle (Fig. 2). The nerve was seen to most commonly exit medial to the sartorius muscle with a prevalence of $86.8 \%$ (95\% CI 71.7-90.0). All other exit patterns were seen quite rarely, with rarest of them being over the IL, which had a pooled prevalence of only $0.9 \%$ (95\% CI 0.0-3.6). Forest plots of the exits of the LFCN are presented in Supplement 2.

Subgroup analysis by geography revealed comparable results in all continents with an exit medial to the sartorius muscle being most common in all populations. However, it was seen slightly more commonly in South Americans with a pooled prevalence of $88.5 \%$ (95 \% CI 63.0-100.0) and less commonly in Europeans with a pooled prevalence of $81.1 \%$ (95\% CI 58.4-92.4). 
Table 2 Characteristics of included studies

\begin{tabular}{|c|c|c|c|}
\hline References & Country & Type of study & $n$ (number of lower limbs) \\
\hline Zhao et al. [32] & China & Cadaveric & 100 \\
\hline Aszmann et al. [6] & USA & Cadaveric & 104 \\
\hline Sürücü et al. [3] & Turkey & Cadaveric & 37 \\
\hline De Ridder et al. [14] & Netherlands & Cadaveric & 116 \\
\hline Hospodar et al. [15] & USA & Cadaveric & 67 \\
\hline Murata et al. [16] & Japan & Cadaveric & 205 \\
\hline Da Rocha et al. [17] & Brazil & Cadaveric & 20 \\
\hline Dias Filho et al. [18] & Brazil & Cadaveric & 50 \\
\hline Grothaus et al. [19] & USA & Cadaveric & 29 \\
\hline Mischkowski et al. [20] & Germany & Cadaveric & 34 \\
\hline Shin et al. [21] & Korea & Cadaveric & 12 \\
\hline Bjurlin et al. [22] & USA & Cadaveric & 22 \\
\hline Anloague and Huijbregts [1] & USA & Cadaveric & 34 \\
\hline Damarey et al. [13] & France & Cadaveric & 10 \\
\hline Damarey et al. $[13]^{\mathrm{a}}$ & France & Imaging (ultrasound) & 26 \\
\hline Doklamyai et al. [23] & Thailand & Cadaveric & 85 \\
\hline Ropars et al. [24] & France & Cadaveric & 34 \\
\hline Bodner et al. [10] & United Kingdom & Imaging (ultrasound) & 17 \\
\hline Kosiyatrakul et al. [29] & Thailand & Cadaveric & 96 \\
\hline Majkrzak et al. [30] & USA & Cadaveric & 65 \\
\hline Ray et al. [25] & India & Cadaveric & 47 \\
\hline Martins et al. [26] & Brazil & Cadaveric & 120 \\
\hline Üzel et al. [27] & Turkey and Germany & Cadaveric & 42 \\
\hline Zhu et al. [11] & China & Imaging (ultrasound) & 240 \\
\hline Watson et al. [12] & USA & Imaging (MRI) & 100 \\
\hline Chowdhry et al. [31] & USA & Cadaveric & 50 \\
\hline Reinpold et al. [28] & Germany & Cadaveric & 58 \\
\hline
\end{tabular}

a Same study as the one above it, however two populations/modalities were employed and considered separate

A sensitivity analysis was performed by including only studies $>100$ lower limbs to probe the sources of heterogeneity. Our analysis revealed results, which were not statistically significant and were comparable to results of our overall analysis. Further data on the exit of the LFCN in the pelvis is reported in Table 3 .

\section{Branching patterns of the lateral femoral cutaneous nerve}

Fourteen studies ( $n=993$ lower limbs) reported data on the various branching patterns of the LFCN in the inguinal region. These patterns include no branching in the area of the IL, bifurcation within the pelvis, bifurcation in the area of the IL, trifurcation and quadrification of the LFCN. The LFCN most commonly demonstrated the no bifurcation pattern with a prevalence of $79.1 \%$ (95 \% CI 58.7-85.0), followed by bifurcation within the pelvis with a prevalence of $11.8 \%$ (95\% CI 3.1-21.9). The least common branching pattern of quadrification is seen only in $1.0 \%$
(95\% CI 0.0-4.8) of cases. Forest plots of the branching patterns of the LFCN are presented in Supplement 3.

Subgroup analysis by geography also demonstrated a lack of branching of the LFCN to be most common. However, it was more commonly seen in North Americans with a prevalence of $87.8 \%$ (95\% CI 79.6-95.9) and was seen least commonly in South Americans and Europeans who had pooled prevalences of $67.7 \%(95 \%$ CI 27.0-96.6) and 67.2\% (95\% CI 23.7-95.8), respectively.

Sensitivity analysis performed using studies with only 100 or more lower limbs revealed results that were consistent with the overall analysis. Further data on the branching patterns of the LFCN can be found in Table 4 .

\section{Distance from the point of exit of the lateral femoral cutaneous nerve to the anterior superior iliac spine}

Eighteen articles ( $n=1,099$ lower limbs) reported morphometric data on the distance from the point of exit of the LFCN to the ASIS in cases when the LFCN passed medial 


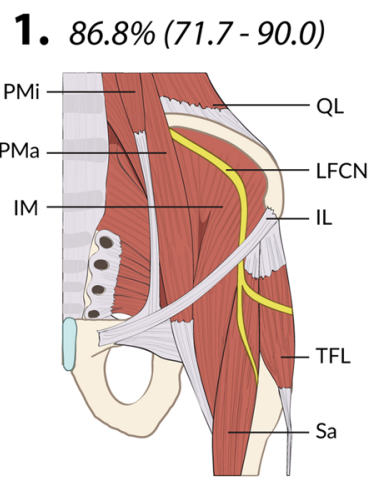

5. $2.6 \%(0.0-6.7)$
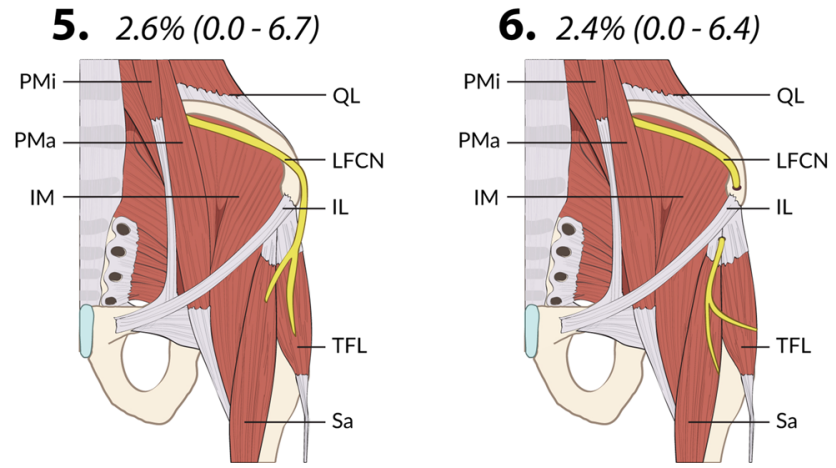

Fig. 2 Types of exits of the lateral femoral cutaneous nerve from the pelvis with calculated pooled prevalence. Type 1 medial to the sartorius (under the IL and medial to the ASIS), type 2 through the IL, type 3 over the IL, type 4 over the ASIS, type 5 lateral (or behind) the ASIS, type 6 through the ASIS, type 7 through the sartorius. All data

to the ASIS and under the IL (the most common exit pattern). The pooled mean distance was found to be $1.90 \mathrm{~cm}$ (95\% CI 1.65-2.14).

Subgroup analysis by geography revealed that Europeans and North Americans had a longer distance between the LFCN exit and the ASIS with pooled mean distances of $2.32 \mathrm{~cm}$ (95\% CI $1.83-2.81)$ and $2.31 \mathrm{~cm}$ (95\% CI 1.54-3.09), respectively, whereas South Americans had the shortest distance of only $0.99 \mathrm{~cm}$ (95\% CI $0.43-1.55)$. Additional subgroup analysis by side revealed comparable distances on the left and right sides. Further data on the pooled mean distance from the point of exit of the LFCN to the ASIS is reported in Table 5.

\section{Discussion}

The variations in the anatomy of the LFCN have been known and documented for many years, with the injury to the LFCN noted as far back as 1885 [6]. The goal of our study was to collect all data available on the variations in the anatomy of the LFCN, namely its points of exit in the pelvis, branching patterns, and distance from other major structures, to provide a better understanding for surgeons operating in its vicinity.

\section{3. $0.9 \%(0.0-3.6)$}
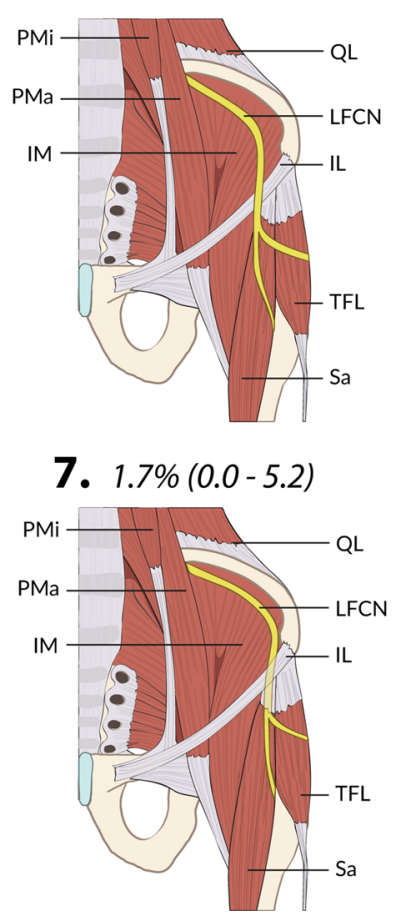

4. $1.9 \%(0.0-5.6)$

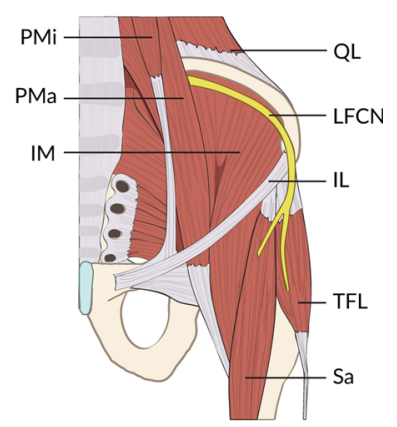

reported as pooled prevalence in percentage with $95 \%$ confidence intervals. $P M a$ psoas major, $P M i$ psoas minor, $I M$ iliacus muscle, $S a$ sartorius, $T F L$ tensor fasciae latae, $I L$ inguinal ligament, $L F C N$ lateral femoral cutaneous nerve, ASIS anterior superior iliac spine, $Q L$ quadratus lumborum

Our analysis showed that a majority of nerves follow the pattern of exiting the pelvis anterior to the ASIS, under the IL and medial to the sartorius muscle, with an overall prevalence of $86.8 \%$, with subgroup analysis showing prevalences above $80 \%$ for all groups. When the nerve exited following this pattern, medial to the ASIS and under the IL, it was usually found $1.9 \mathrm{~cm}$ medial to the ASIS.

The nerve usually exited as a single nerve, with an overall prevalence of $79.1 \%$. Bifurcation within the pelvis was the second most common pattern with a prevalence of $11.8 \%$. It was noted, however, that in studies from South America, there was a much higher prevalence of trifurcation than bifurcation in the pelvis, with a prevalence of 24.7 versus $1.2 \%$, respectively.

The most common pathology described pertaining to the LFCN is meralgia paresthetica, or pain and/or dysesthesia in the area of the lateral thigh that the nerve supplies [2]. The etiology of this pathology can be entrapment of the nerve caused by everything from physiological changes in the inguinal area, to the clothes a person wears. It is important to note that iatrogenic injury during surgery is also a common cause. Therefore, proper knowledge of the possible variations in the anatomy of the LFCN is important in the planning and execution of surgery in the vicinity of the LFCN. 

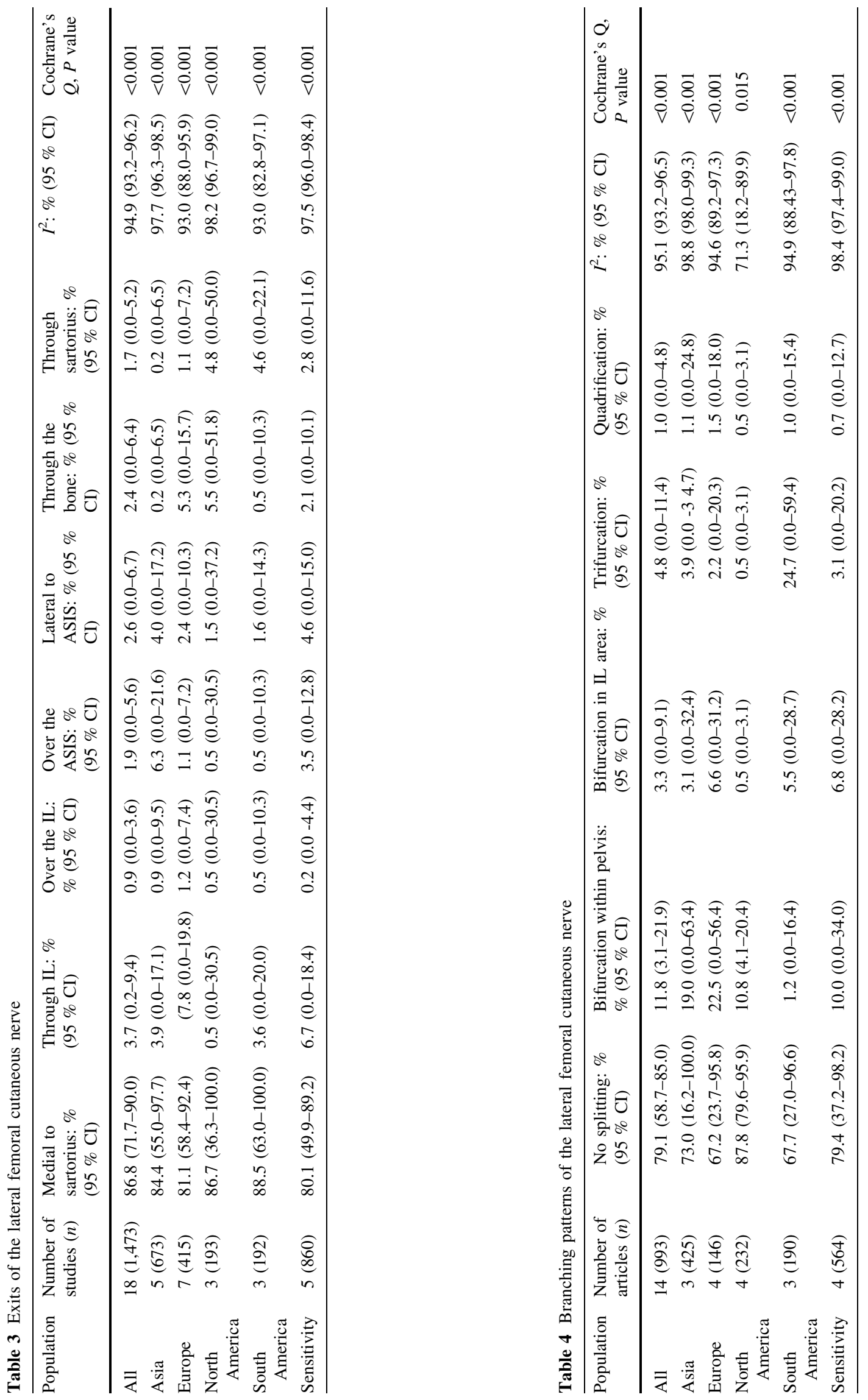
Table 5 Distance from the exit of the lateral femoral cutaneous nerve to the anterior superior iliac spine

\begin{tabular}{lclc}
\hline Population & Number of articles (number of limbs) & Pooled mean $(\mathrm{cm}):(95 \% \mathrm{CI})$ & $I^{2}: \%$ \\
\hline All & $18(1,099)$ & $1.90(1.65-2.14)$ & 52.24 \\
Asia & $5(459)$ & $1.43(0.98-1.89)$ & 0.00 \\
Europe & $6(166)$ & $2.32(1.83-2.81)$ & 0.00 \\
North America & $5(304)$ & $2.31(1.54-3.09)$ & 0.00 \\
South America & $2(170)$ & $0.99(0.43-1.55)$ & 0.00 \\
Left & $5(201)$ & $1.99(1.44-2.53)$ & 35.49 \\
Right & $5(110)$ & $1.91(1.38-2.43)$ & 28.55 \\
Sensitivity & $15(639)$ & $2.00(1.63-2.38)$ & $21.67 \%$ \\
\hline
\end{tabular}

Consideration of the variations in the LFCN are especially important in conducting inguinal hernia repairs. Though rare variations of the nerve travelling through the ASIS, the IL, or through the sartorius muscle may be better protected from superficial injury, the majority of patients' LFCNs are at a risk of iatrogenic injury. Patients with early bifurcations, including those within the pelvis and in the area of the IL would be at higher risk of iatrogenic injury during surgery as there are more branches to keep track of in the area compared to the normal anatomy. Similarly, trifurcations and quadrifications of the LFCN provide more targets for accidental injury, putting populations from regions of South America where trifurcations presented with a prevalence of $24.7 \%$ at an elevated risk.

Clinical data have shown laparoscopic inguinal hernia repair to be a safer alternative to open repair in terms of incidence of postoperative neuralgias with a relative risk ratio of 0.66 (95\% CI 0.51-0.87) when compared to open inguinal hernia repair [33]. When evaluating a laparoscopic approach, it has been suggested that staples be avoided within $1 \mathrm{~cm}$ of the ASIS due to the proximity of the LFCN [34]. In our subgroup analysis by geographical region, we noticed that there was very little heterogeneity for the pooled mean distances of the LFCN from the ASIS. Thus, we suspect the cause for heterogeneity in our overall analysis of distance of the LFCN from the ASIS was most likely due to the geographical differences. Our analysis revealed that South American populations had LFCNs closest to the ASIS with a mean distance of $0.99 \mathrm{~cm}(95 \%$ CI 0.43-1.55). European and North American populations, on the other hand, had LFCN's with mean distances of $2.32 \mathrm{~cm}(95 \%$ CI $1.88-2.81)$ and $2.31 \mathrm{~cm}(95 \% \mathrm{CI}$ 1.54-3.09) from the ASIS, respectively. Asian populations fell in between with a mean distance of $1.43 \mathrm{~cm}(95 \% \mathrm{CI}$ 0.98-1.89). We would like to suggest that the dangerous zone for staples should be re-evaluated due to the fact that our data suggests that the average LFCN will pass within $1.9 \mathrm{~cm}$ of the ASIS and is highly variable depending on where the patient is from. With other procedures, such as aesthetic abdominoplasties, a zone of $4 \mathrm{~cm}$ around the ASIS has been demarcated as a potentially dangerous area requiring careful dissection and preservation, to retain proper LFCN structure and function [31].

Studies have reported that the general rule of thumb used by surgeons is approximating the LFCN as running two fingerbreadths medial to the ASIS [30]. Such a strategy however, can grossly miscalculate the location of the nerve depending on the patient as well as the surgeon's anatomical knowledge. Ideally an imaging approach like ultrasound would help to determine the precise location of the LFCN and confirm that one of the other common variations is not present. However, if a gross estimate must be made, we would suggest $3 \mathrm{~cm}$ as a rule of thumb, rather than simply two fingerbreadths, as finger width can vary among the population. Based on our analysis, we ideally suggest a danger zone for all surgical procedures of about $3 \mathrm{~cm}$ around the ASIS, which corresponds to the upper limit of the confidence interval of the subgroup with the highest upper limit in the confidence interval (North America), and thus minimizing the risk of iatrogenic injury for the majority of the population.

Another procedure where the location of the LFCN is of particular interest is bone graft harvesting. Size of the graft, and size of incision can greatly influence the risk of injury. The current suggestions are that the grafts should be $<3 \mathrm{~cm}$ in size and that the incisions being made should be at least $3 \mathrm{~cm}$ or more away from the palpable point of the ASIS [29]. This general guideline could potentially injure patients with an LFCN that is lateral to the ASIS, which was found in $2.6 \%$ of the population studied $(95 \% \mathrm{CI}$ 0.0-6.7). Thus we would recommend an imaging study like ultrasound before graft sampling.

A final consideration of the LFCN is for the anterior approach to hip arthroplasty. In a study in $2010,81 \%$ of patients reported new onset of neurapraxia in the area supplied by the LFCN after a hip resurfacing or total hip arthroplasty performed using the anterior approach [35]. The anterior approach offers many advantages over the posterior approach, which has a higher risk of dislocation, and the lateral approach, which puts the adduction function at risk [36]. With the anterior approach offering the least damage to a patient's hip function, the loss of sensation 
provided by the LFCN becomes a larger concern. Current suggestions for minimally invasive anterior approaches suggest incisions running parallel to the LFCN [37]. Again, in order for this approach to be viable and the LFCN preserved, the location of the LFCN must be strictly determined, not simply estimated due to the high variability.

Our meta-analysis was limited by the variety of ways individual studies assess the anatomy of the LFCN. Though most studies follow a general pattern, new imaging studies and three-dimensional imaging may lead to some interpretation problems when comparing to older studies' measured values. Additionally, high heterogeneity between studies, and a lack of assessment of publication bias due to a lack of statistical measure for multi-categorical prevalence, were limiting factors. According to our analysis, the clearest source of heterogeneity for measurements of the LFCN from the ASIS was geographical distribution. Whenever possible, authors were contacted for clarification and a consensus was reached with the research team to minimize bias in the collection and analysis.

We suggest further analysis of the LFCN and its variations, especially with the use of USG as a quick and effective method, to help surgeons minimize the incidence of meralgia paresthetica due to iatrogenic injury to the LFCN.

\section{Conclusion}

Current techniques and planning of safe zones for surgeries in the area of the pelvis are close estimates to the normal anatomy of the LFCN. Though the normal textbook anatomy describes the LFCN as passing out of the pelvis as a single nerve, under the IL and medial to the ASIS, countless variations are commonly encountered. Therefore, a proper understanding and re-evaluation of individual patients' anatomy is crucial for making proper safe zones for surgery. Ideally, we would like to suggest considering the area within $3 \mathrm{~cm}$ from the ASIS as a danger zone in all surgical procedures. These variations, combined with the number of surgeries performed in the inguinal and hip area, namely inguinal hernia repair and hip replacement procedures, become significant in the prevention of meralgia paresthetica and iatrogenic injury. Proper knowledge of the variations possible can reduce the risk of nerve damage and improve the outcomes of procedures like inguinal hernia repair, hip arthroplasty, abdominoplasty, and iliac bone grafting.

Acknowledgments Krzysztof A. Tomaszewski was supported by the Foundation for Polish Science (FNP). We would like to thank Karolina Saganiak for the anatomical drawings used in this manuscript. This study was funded by statutory grants of the Jagiellonian University Medical College. The sponsors did not play any role in the study design; in the collection, analysis and interpretation of data; in the writing of the report; and in the decision to submit the article for publication.

Compliance with ethical standards This study complies with the current laws of the country in which they were performed.

Conflict of interest Krzysztof A. Tomaszewski, Patrick Popieluszko, Brandon Michael Henry, Joyeeta Roy, Beatrice Sanna, Mark Robert Kijek, and Jerzy A. Walocha declare that they have no conflict of interest.

Research involving human participants and/or animals This article does not contain any studies with human participants or animals performed by any of the authors.

Informed consent This article does not require informed consent due to the lack of human participants.

Open Access This article is distributed under the terms of the Creative Commons Attribution-NonCommercial 4.0 International License (http://creativecommons.org/licenses/by-nc/4.0/), which permits any noncommercial use, distribution, and reproduction in any medium, provided you give appropriate credit to the original author(s) and the source, provide a link to the Creative Commons license, and indicate if changes were made.

\section{References}

1. Anloague PA, Huijbregts P (2009) Anatomical variations of the lumbar plexus: a descriptive anatomy study with proposed clinical implications. J Man Manip Ther 17:107-114

2. Carai A, Fenu G, Sechi E et al (2009) Anatomical variability of the lateral femoral cutaneous nerve: findings from a surgical series. Clin Anat 22:365-370

3. Sürücü HS, Tanyeli E, Sargon MF, Karahan ST (1997) An anatomic study of the lateral femoral cutaneous nerve. Surg Radiol Anat 19:307-310

4. Rosenberg J, Bisgaard T, Kehlet $\mathrm{H}$ et al (2011) Danish Hernia Database recommendations for the management of inguinal and femoral hernia in adults. Dan Med Bull 58:C4243

5. Bay-Nielsen M, Kehlet H, Strand L et al (2001) Quality assessment of 26,304 herniorrhaphies in Denmark: a prospective nationwide study. Lancet 358:1124-1128

6. Aszmann OC, Dellon ES, Dellon AL (1997) Anatomical course of the lateral femoral cutaneous nerve and its susceptibility to compression and injury. Plast Reconstr Surg 100:600-604

7. Moher D, Liberati A, Tetzlaff J, Altman DG (2009) Preferred reporting items for systematic reviews and meta-analyses: the PRISMA statement. PLoS Med 6(7):e1000097

8. Henry BM, Zwinczewska H, Roy J et al (2015) The prevalence of anatomical variations of the median nerve in the carpal tunnel: a systematic review and meta-analysis. PLoS One 10:e0136477

9. Higgins J, Green S (2011) Cochrane handbook for systematic reviews of interventions. Version 5.1.0. The Cochrane Collaboration. http://cochrane-handbook.org

10. Bodner G, Bernathova M, Galiano K et al (2009) Ultrasound of the lateral femoral cutaneous nerve: normal findings in a cadaver and in volunteers. Reg Anesth Pain Med 34:265-268

11. Zhu J, Zhao Y, Liu F et al (2012) Ultrasound of the lateral femoral cutaneous nerve in asymptomatic adults. BMC Musculoskelet Disord 13:227 
12. Watson JN, Bohnenkamp F, El-Bitar Y et al (2014) Variability in locations of hip neurovascular structures and their proximity to hip arthroscopic portals. Arthroscopy 30:462-467

13. Damarey B, Demondion X, Boutry N et al (2009) Sonographic assessment of the lateral femoral cutaneous nerve. J Clin Ultrasound 37:89-95

14. De Ridder VA, De Lange S, Popta J (1999) Anatomical variations of the lateral femoral cutaneous nerve and the consequences for surgery. J Orthop Trauma 13:207-211

15. Hospodar PP, Ashman ES, Traub JA (1999) Anatomic study of the lateral femoral cutaneous nerve with respect to the ilioinguinal surgical dissection. J Orthop Trauma 13:17-19

16. Murata Y, Takahashi K, Yamagata M et al (2000) The anatomy of the lateral femoral cutaneous nerve, with special reference to the harvesting of iliac bone graft. J Bone Jt Surg Am 82A:746-747

17. Da Rocha RP, Fernandes GJM, Vengjer A et al (2002) Distribution of the lateral cutaneous nerve of the thigh in the area of intramuscular injection. Rev Assoc Med Bras 48:353-356

18. Dias Filho LC, Valença MM, Guimarães Filho FAV et al (2003) Lateral femoral cutaneous neuralgia: an anatomical insight. Clin Anat 16:309-316

19. Grothaus MC, Holt M, Mekhail AO et al (2005) Lateral femoral cutaneous nerve: an anatomic study. Clin Orthop Relat Res 437:164-168

20. Mischkowski RA, Selbach I, Neugebauer J et al (2006) Lateral femoral cutaneous nerve and iliac crest bone grafts-anatomical and clinical considerations. Int $\mathrm{J}$ Oral Maxillofac Surg 35:366-372

21. Shin YB, Park JH, Kwon DR, Park BK (2006) Variability in conduction of the lateral femoral cutaneous nerve. Muscle Nerve 33:645-649

22. Bjurlin MA, Davis KE, Allin EF, Ibrahim DT (2007) Anatomic variations in the lateral femoral cutaneous nerve with respect to pediatric hip surgery. Am J Orthop (Belle Mead NJ) 36:143-146

23. Doklamyai P, Agthong S, Chentanez V et al (2008) Anatomy of the lateral femoral cutaneous nerve related to inguinal ligament, adjacent bony landmarks, and femoral artery. Clin Anat 21:769-774

24. Ropars M, Morandi X, Huten D et al (2009) Anatomical study of the lateral femoral cutaneous nerve with special reference to minimally invasive anterior approach for total hip replacement. Surg Radiol Anat 31:199-204
25. Ray B, D'Souza AS, Kumar B et al (2010) Variations in the course and microanatomical study of the lateral femoral cutaneous nerve and its clinical importance. Clin Anat 23:978-984

26. Martins RS, Siqueira MG, Silva FC et al (2011) A practical approach to the lateral cutaneous nerve of the thigh: an anatomical study. Clin Neurol Neurosurg 113:868-871

27. Üzel M, Akkin SM, Tanyeli E, Koebke J (2011) Relationships of the lateral femoral cutaneous nerve to bony landmarks. Clin Orthop Relat Res 469:2605-2611

28. Reinpold W, Schroeder AD, Schroeder M et al (2015) Retroperitoneal anatomy of the iliohypogastric, ilioinguinal, genitofemoral, and lateral femoral cutaneous nerve: consequences for prevention and treatment of chronic inguinodynia. Hernia 19:539-548

29. Kosiyatrakul A, Nuansalee N, Luenam S et al (2010) The anatomical variation of the lateral femoral cutaneous nerve in relation to the anterior superior iliac spine and the iliac crest. Musculoskelet Surg 94:17-20

30. Majkrzak A, Johnston J, Kacey D, Zeller J (2010) Variability of the lateral femoral cutaneous nerve: an anatomic basis for planning safe surgical approaches. Clin Anat 23:304-311

31. Chowdhry S, Davis J, Boyd T et al (2015) Safe tummy tuck: anatomy and strategy to avoid injury to the lateral femoral cutaneous nerve during abdominoplasty. Eplasty 15:e22

32. Zhao S, Qui L, Di P et al (1995) The anatomy of the lateral cutaneous nerve of the thigh-a possible donor nerve for facial nerve repair. Int J Oral Maxillofac Surg 24:245-247

33. O'Reilly EA, Burke JP, O'Connell PR (2012) A meta-analysis of surgical morbidity and recurrence after laparoscopic and open repair of primary unilateral inguinal hernia. Ann Surg 255:846-853

34. Dibenedetto LM, Lei Q, Gilroy AM et al (2009) Variations in the inferior pelvic pathway of the lateral femoral cutaneous nerve: implications for laparoscopic hernia repair. Clin Anat 9:232-236

35. Goulding K, Beaulé PE, Kim PR, Fazekas A (2010) Incidence of lateral femoral cutaneous nerve neuropraxia after anterior approach hip arthroplasty. Clin Orthop Relat Res 468:2397-2404

36. Bhargava T, Goytia RN, Jones LC, Hungerford MW (2010) Lateral femoral cutaneous nerve impairment after direct anterior approach for total hip arthroplasty. Orthopedics 33:472

37. Jameson SS, Howcroft DWJ, McCaskie AW, Gerrand CH (2008) Injury to the lateral femoral cutaneous nerve during minimally invasive hip surgery: a cadaver study. Ann R Coll Surg Engl 90:216-220 